\title{
Gating, enhanced gating, and beyond: information utilization strategies for motion management, applied to preclinical PET
}

\author{
Adam Leon Kesner ${ }^{1 *}$, Galith Abourbeh ${ }^{2}$, Eyal Mishani ${ }^{2}$, Roland Chisin ${ }^{1}$, Sagi Tshori ${ }^{1}$ and Nanette Freedman ${ }^{1}$
}

\begin{abstract}
Background: Respiratory gating and gate optimization strategies present solutions for overcoming image degradation caused by respiratory motion in PET and traditionally utilize hardware systems and/or employ complex processing algorithms. In this work, we aimed to advance recently emerging data-driven gating methods and introduce a new strategy for optimizing the four-dimensional data based on information contained in that data. These algorithms are combined to form an automated motion correction workflow.

Methods: Software-based gating methods were applied to a nonspecific population of 84 small-animal rat PET scans to create respiratory gated images. The gated PET images were then optimized using an algorithm we introduce as 'gating+' to reduce noise and optimize signal; the technique was also tested using simulations. Gating + is based on a principle of only using gated information if and where it adds a net benefit, as evaluated in temporal frequency space. Motion-corrected images were assessed quantitatively and qualitatively.

Results: Of the small-animal PET scans, 71\% exhibited quantifiable motion after software gating. The mean liver displacement was $3.25 \mathrm{~mm}$ for gated and $3.04 \mathrm{~mm}$ for gating+ images. The (relative) mean percent standard deviations measured in background ROls were 1.53, 1.05, and 1.00 for the gated, gating+, and ungated values, respectively. Simulations confirmed that gating + image voxels had a higher probability of being accurate relative to the corresponding ungated values under varying noise and motion scenarios. Additionally, we found motion mapping and phase decoupling models that readily extend from gating+ processing.

Conclusions: Raw PET data contain information about motion that is not currently utilized. In our work, we showed that through automated processing of standard (ungated) PET acquisitions, (motion-) information-rich images can be constructed with minimal risk of noise introduction. Such methods have the potential for implementation with current PET technology in a robust and reproducible way.
\end{abstract}

Keywords: Motion control, Software-based gating, Hardware-based gating, Gating+, Inter-gate values, Small-animal PET

\section{Background}

In the evolution of nuclear medicine imaging technologies, there have been steady advancements towards better sensitivity and resolution. Resolution has in fact improved so much that for some parts of the body, further improvements will be of no help as respiratory and cardiac motions limit the benefits. Respiratory and cardiac motions

\footnotetext{
* Correspondence: adam.kesner@fulbrightmail.org

'Department of Medical Biophysics and Nuclear Medicine, Kiryat Hadassah, P.O. Box 12000, Jerusalem 91120, Israel

Full list of author information is available at the end of the article
}

cause blurring in imaging, particularly around the lungs and diaphragm [1,2]. Image degradation may include poorer lesion detectability and inaccuracy in location, volume definition, and quantitation. Gating may be used to overcome these problems and realize the benefits of highresolution imaging [3-5].

Respiratory gating has been studied for over a decade in PET imaging, both in small-animal PET [6] and human PET $[7,8]$. Many commercial systems today include hardware devices, e.g., a pressure belt [9-11], motion camera $[8,12]$, or other systems $[13,14]$, to monitor respiratory 
signal, along with gating software to process it. However, in the past few years, several data-driven algorithms have been presented for extracting respiratory signal directly from the raw scan data without using hardware. These algorithms perform comparably to hardware $[12,15]$, can be fully automated [16-19], and can be used with no changes to current clinical scanning procedures.

Software-based algorithms can offer advantages over hardware-based gating, both in preclinical and clinical environments. Since they are based solely on analysis of image data and not hardware equipment, they can be used with existing scanners. In contrast to hardware systems, they avoid a possible source of subject discomfort, costly equipment, and potential for equipment failure. They require no additional scan setup time or staff training and avert higher radiation doses to patients and technologists from the added operations/slower throughput [20]. Such methods are operator independent and reproducible, and the gating signals are intrinsically aligned with the image data.

Beyond gated image acquisition, questions arise as to how to best use the data. While conventional PET produces summed images of all phases of the respiratory cycle, gating generates a series of images at different phases of the cycle. These gated images have improved spatial resolution since each image includes only a short phase of the respiratory cycle, but each image also includes fewer counts than the summed ungated image and, thus, is noisier. Because of this inherent trade-off between noise and resolution, it can be difficult for the human eye, or even computer-aided systems, to distinguish between added value (e.g., organ edges, motion) and misleading information introduced by noise effects [10].

Techniques have been developed to address the sacrifice in statistics inherent in gating. While strategies for optimized data binning have been presented $[11,21]$, the primary efforts for full data utilization use nonlinear deformation maps to map information from different gates to a target gate, essentially recombining the gates back into a single motion-free frame with high resolution and high count statistics [22-24]. Limitations of these techniques are that they can be complex, parameter and distribution dependent, prone to error, difficult to fully characterize, and some require heavy processing $[25,26]$.

A very different approach used previously to reduce noise in gated nuclear medicine data is through filtering. Temporal frequency filters can be applied to dynamic or gated data to produce less noisy images [27]. Filtering offers a computationally easy method for reducing noise but has not been adopted for larger-scale use. One problem of the technique is that filtering may improve the accuracy in some pixels but not in others. Voxel-specific filtering, based on the noise and signal characteristics of each individual voxel, has the potential to avoid this problem [28].
In recent years, methods based on strategies of randomly sorting or bootstrapping data have been developed to estimate effective gate-specific, voxel-specific noise in gated data [29]. This approach provides a measure of effective noise, essentially independent of the processing routines used to create the image. The method we propose in this paper utilizes this concept for noise estimation and combines it with previously presented ideas for noise filtering as a foundation for developing an advanced filtering technique for optimizing information in the fourdimensional (4D) (gated) signal.

In the work presented here, we aim to extend and improve a strategy for creating software-gated images, previously applied to human PET, to small-animal PET using a population of scans acquired with a variety of radiotracers. In addition, we present a new voxel-based filtering approach, which we denote as 'gating+', to address the problem of low statistics in subsampled gated images and to enable clear visualization of image features in the presence of respiratory motion. Validation is performed using simulations as well as the small-animal PET scans.

\section{Methods}

Software-based respiratory signal was extracted from the raw listmode files of 84 rat PET scans. The data consisted of all scans with duration of at least 10 min acquired during research studies at our institution over a period of 12 months. All studies were approved by the Animal Research Ethics Committee of the Hebrew University of Jerusalem. Tracers utilized included ${ }^{18} \mathrm{~F}$-fluorodeoxyglucose $\left({ }^{18} \mathrm{~F}\right.$-FDG, $\left.n=27\right)$, ${ }^{11} \mathrm{C}$-dimethyl-diphenyl-ammonium $\left({ }^{11} \mathrm{C}\right.$-DMDPA, $\left.n=10\right)$ $[30],{ }^{13} \mathrm{~N}-\mathrm{NH}_{3}(n=5),{ }^{11} \mathrm{C}$-choline $(n=2),{ }^{18} \mathrm{~F}-\mathrm{NaF}(n=1)$, ${ }^{18} \mathrm{~F}$-fluoroethyl-diphenyl-methyl-ammonium $\left({ }^{18} \mathrm{~F}\right.$-FEDPMA, $n=12$ ) and ${ }^{18} \mathrm{~F}$-fluorobuthyl-diphenyl-methyl-ammonium $\left({ }^{18} \mathrm{~F}\right.$-FBDPMA, $\left.n=4\right)\left({ }^{18} \mathrm{~F}\right.$-FEDPMA and ${ }^{18} \mathrm{~F}$-FBDPMA are investigational new compounds for PET myocardial perfusion imaging), and ${ }^{18} \mathrm{~F}-\mathrm{ML} 10(n=23$, agent for imaging apoptosis [31]). Twenty four of the scans (11 ${ }^{18} \mathrm{~F}-\mathrm{FDG}, 5$ ${ }^{11}$ C-DMDPA, $4{ }^{18} \mathrm{~F}$-FEDPMA, and $4{ }^{18}$ F-FBDPMA) were acquired with hardware-based respiratory gating, and for this subset of scans, hardware-based signals were compared with the corresponding software-based signal.

\section{Scan acquisition}

All scans were acquired using a Siemens Inveon smallanimal PET scanner (Siemens Healthcare ${ }^{\circ}$ Knoxville, TN, USA). Scans were reconstructed, using three-dimensional (3D) sinograms and OSEM2D reconstruction (4 iterations and 16 subsets), into $128 \times 128 \times 159$ images with a voxel size of $0.7764 \times 0.7764 \times 0.796 \mathrm{~mm}^{3}$. All images were smoothed with a $2-\mathrm{mm}^{3}$ full width at half maximum (FWHM) Gaussian smoothing filter. Random correction was performed by subtraction of delayed coincidences. No attenuation or scatter corrections were used. For the 24 
scans acquired with hardware-based gating, the gating signal was acquired using a Biovet ${ }^{\circ}$ gating system (Biovet ${ }^{\circ}$, M2Mimaging, Cleveland, OH, USA). This system acquires respiratory signal through the use of a pressure-sensitive pad placed beneath the rat.

The duration of scanning differed among the research studies. For the sake of uniformity, only $10 \mathrm{~min}$ of each scan was analyzed. The 84 scans were acquired using rats of varying strains and sizes (100 to $500 \mathrm{~g}$ ) in prone position, with injected doses ranging between $300 \mu \mathrm{Ci}$ and $2 \mathrm{mCi}$, and total detected prompts ranging between $4.8 \times 10^{6}$ and $7.9 \times 10^{8}$ counts (over $600 \mathrm{~s}$ ). The part of the animal included in the $126.6-\mathrm{mm}$ axial field of view (FOV) varied since the 84 scans were from diverse studies, with organs of interest ranging from the brain, the heart, the lungs, or leg muscles; thus, while most scans included all or almost all of the thorax and abdomen, some did not.

\section{Software-based gating procedures}

Listmode files, the initial raw output from a PET scanner, consist of a list of detected events interspersed by time stamps. In the case of hardware gating, the listmode file includes hardware-based gating triggers inserted at relevant time points; these triggers are used during construction of the gated images.

The main steps involved in data-driven gating can be summarized as follows:

Step 1. Respiratory signal is extracted from the listmode data.

Step 2. Respiratory gating triggers, derived from step 1 signal, are inserted to form a new gated listmode file (analogous to the one created with hardware-based triggers).

Step 3. Gated images are reconstructed from the new listmode file.

The software-based gating method has been described previously for application in human PET $[18,19]$. As a subject breathes, the activity concentration in fixed regions of space fluctuates with frequencies corresponding to respiration. The signal in each of these regions is small and noisy, but by combining the fluctuating signal in many regions, we can extract a useful 'global' respiratory signal to be used for gating. The algorithm was implemented as described previously but with relevant parameters adjusted for differences across the technologies/species. The average breathing frequencies for humans and rats are approximately 0.2 and $1 \mathrm{~Hz}$, respectively. Accordingly, the frequency pass window we used here for voxel prioritization and combination was 0.66 to $3.33 \mathrm{~Hz}$. The time bin parameter duration of the short time sinograms used for sampling time activity - was $100 \mathrm{~ms}$, approximately one-tenth of an average respiratory cycle [18,32]. Finer sampling times were deemed unnecessary in this step, particularly in light of the fact that the vendor supplied gating software utilized moving averages (over 8 cycles) for respiratory period determination.

In addition to the published methods, one enhancement was introduced: Previously, when the signal from each sinogram region was combined with the global signal, a simple test was applied to see if the signal was in phase or out of phase with the global signal (step 5B in previous article [18]). This same test was presented here, but the entire signal duration was split into multiple (six) equal time segments (i.e., time-activity curves with length of $10 \mathrm{~min} / 6$ ). The test was applied to all six segments separately, and only when all phase tests agreed was the signal of a small sinogram region added to the global respiratory signal. This modification helped exclude data from voxels that were too noisy or did not contain useful information, thus improving the signal-to-noise ratio in the output. The value of six segments was chosen empirically to be great enough to consistently filter out random signals while not so great that it would filter out useful signal. As a clarification, uniform periodicity is not a requisite for this technique to adequately capture respiratory signal.

In our specific implementation, 3D data were binned into two-dimensional (2D) sinograms using single-slice rebinning (SSRB) [33] (a strategy used for data-driven gating presented by Schleyer and colleagues [12]). The SSRB sinograms had dimensions $(\rho, \theta, z)$ of $128 \times 160 \times 159$. The voxels had dimensions $\rho=0.815 \mathrm{~mm}$ and $\theta=\pi \mathrm{rad} / 160$, and the scanner had an axial crystal pitch of $1.592 \mathrm{~mm}$.

In step 2, the one-dimensional (1D) global respiratory trace acquired in step 1 was analyzed to extract respiratory triggers. The trigger points, i.e., times where a new respiratory cycle has begun, were defined as points in time when the $1 \mathrm{D}$ global respiratory trace was at a local maximum $( \pm 1 / 2$ the most represented respiratory period (s)). These respiratory trigger points were then inserted back into the raw listmode files (in the format suitable for our reconstruction software) to create modified listmode files.

In step 3, 4D gated image data sets were generated from the listmode files using the Siemens reconstruction software exactly as they would have been reconstructed in the case of hardware gating. The 4D gated data sets contain information in three spatial dimensions and one temporal (i.e., gated) dimension. In this paper, we will refer to the dimension of information across gates as 'temporal'.

\section{Gating+ algorithm}

Gating+ uses a frequency filter to generate enhanced gated images that include respiratory motion but preserve the 
favorable noise characteristics of the ungated image. The filter passes the ungated image (zero frequency) plus those higher frequencies that add more (constructive) motion information than (destructive) noise. Noise and motion are nonuniformly distributed throughout an image; correspondingly, we used the signal and noise characteristics of each individual voxel to generate a scan-specific and spatially variant band-pass filter.

Voxel-specific noise characteristics are evaluated using a method of random gating. A 4D randomly gated image set is generated by subdividing the original listmode data based on random triggers instead of triggers derived from respiratory motion. The behavior of the resultant images provide an indication of how much noise and fluctuations are to be expected simply from the act of gating, without any motion information confounding the issue. The voxel-specific noise reflects statistical noise in subsampled signal, random fluctuations, and any system noise/bias. Characterized for our processing, a voxel's effective noise magnitude is conservatively defined as the maximum signal amplitude of all nonzero frequencies in the randomly gated data set.

To implement voxel-specific filtering, the fast Fourier transform is first applied to each voxel in both the respiratory gated and the randomly gated data sets in the temporal dimension. This yields two sets of vectors in real and imaginary frequency space, describing both motion signal and effective noise in the case of the respiratory gated data, and effective noise alone in the case of the randomly gated data. The gating+ algorithm compares the vector magnitudes at every frequency to determine the appropriate frequencies to pass effectively those frequencies that make a discernible contribution. Because higher frequencies require greater statistics to support them, the useful-frequency windows span lower frequencies to an upper cutoff frequency. Specifically, a band-pass filter is defined with a lower bound of 0 (direct current (DC) signal), and an upper bound determined as the highest contiguous frequency where the magnitude of the fluctuating signal is sufficiently greater than the magnitude of the noise (>1.2 $\times$ effective noise magnitude for the corresponding voxel in the randomly gated dataset). The gated information in that frequency window is allowed to pass through the filter and thus modify the image from its ungated embodiment (zero frequency image). Signal in frequencies outside this window are filtered, i.e., truncated to 0 . The threshold of 1.2 was derived from Monte Carlo simulations which address the fact that noise presents with an unknown/random phase (see 'Discussion' section for further details). This value represents the probability threshold where using the gated signal fluctuations becomes advantageous $(P(1.2)=$ $0.5)$. After all voxels are processed, gating + images are obtained by performing the inverse fast Fourier transform on the filtered data to yield a set of enhanced gated images. A flowchart illustrating the gating+ process is shown in Figure 1.

\section{Motion maps and inter-gate phase shifting}

In addition to optimizing the signal, the filtering of noise in the temporal domain also provides two interesting opportunities. Firstly, the frequency pass map, constructed during gating+ filtering, can provide an overview of the detected motion - a 'motion map'. Secondly, because signal is optimized in frequency space where it is not bound to gates it was created with, there is a particular opportunity to manipulate its real and imaginary components to achieve a phase shift when it is transformed back into image space, essentially allowing us to extract inter-gate voxel values (demonstrated in Figure 2). By uniformly shifting all voxels, we can reconstruct phase-shifted images that may correspond to any or all phases of the motion cycle. In the work here, we used this process to create 'continuous motion image' (CMI) sequences with finely timed frames that span the motion cycle. The CMIs offer an alternative visualization of motion and may present a new platform for assessing 4D data.

\section{Validation}

To assess the accuracy of gating+ images, simulations were generated, consisting of $2 \mathrm{D}+$ time images containing a hot lesion set in a colder background moving adjacent to a stationary two-compartment structure, with varying amounts of random noise added to simulate environments of varying signal statistics. For the PET data, each listmode file was used to reconstruct an ungated image, gated images, gating+ images, motion map, and CMIs. All images were reconstructed with 16 gates and CMIs with 90 frames. The large number of gates was chosen because, in contrast to gating, gating+ images appeared to benefit from having more gates, i.e., more available frequencies to utilize when statistics support it.

Gated PET images were rendered in the form of maximum-intensity projection image sequences for qualitative review by four independent reviewers who evaluated the scans for presence of 'obvious characteristics of respiratory motion'. For quantitative comparison of hardware-gated, software-gated, and gating+ images, we used the global center of mass (COM) displacement as a general measure of motion.

Many aspects of respiratory motion will shift the global activity COM during the respiratory cycle, although it is acknowledged that some do not. The COM displacement between gates appeared to provide a useful, albeit imperfect, measure of respiratory motion that can 


\section{Gating+ flowchart}

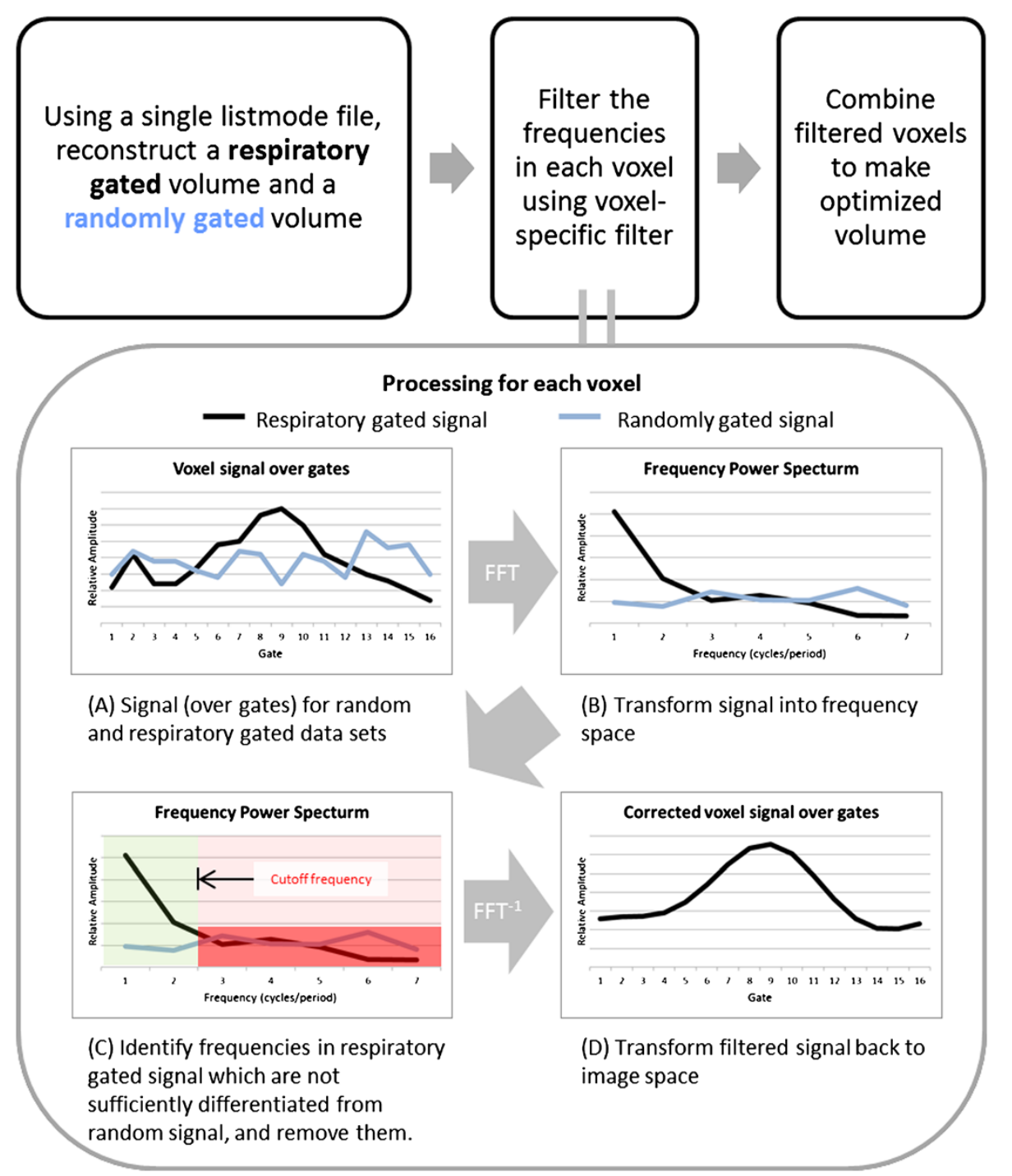

Figure 1 Flowchart illustrating gating+ workflow. The processing applied to each voxel is shown in the lower box. 'Respiratory gated signal' and 'randomly gated signal' refer to the signals for the same voxel in the respiratory motion gated and randomly gated datasets, respectively.

be applied to any image. The COM was calculated for each gate (Equation 1):

$$
x_{\mathrm{cm}}=\frac{\sum_{\mathrm{i}} m_{\mathrm{i}} x_{\mathrm{i}}}{M} \quad y_{\mathrm{cm}}=\frac{\sum_{\mathrm{i}} m_{\mathrm{i}} y_{\mathrm{i}}}{M} \quad z_{\mathrm{cm}}=\frac{\sum_{\mathrm{i}} m_{\mathrm{i}} z_{\mathrm{i}}}{M}
$$

where $m_{\mathrm{i}}$ is the summed voxel intensity for the image plane, $\Sigma_{\mathrm{i}}$ is the sum of all image planes in the respective dimensions, $x_{\mathrm{i}}, y_{\mathrm{i}}$, and $z_{\mathrm{i}}$ are the coordinates of the image planes, $M$ is the total activity in the images, and $x_{\mathrm{cm}}$, $y_{\mathrm{cm}}$, and $z_{\mathrm{cm}}$ are the coordinates of the COM. The maximum COM displacement was calculated as the greatest distance (magnitude of the displacement vector) between any two COMs from different gates. Maximum COM displacement was calculated for all hardware-gated, software-gated, and gating+ scans. In order to justify its validity with respect to our data, we also calculated for global COM measurements for the randomly gated scan set for comparison.

In addition to tracking motion using the COM, we also used volume of interest (VOI) and line profile analysis for those scans that had certain definable characteristics. For scans with uptake in the kidneys, 0.016-cc (33 voxels) spherical VOIs were placed at each kidney centered on the maximum-intensity pixel of an additionally smoothed image (Gaussian smoothing kernel, FWHM $=0.5 \mathrm{~cm}^{3}$ ). For scans with uptake in the liver, line profiles were 


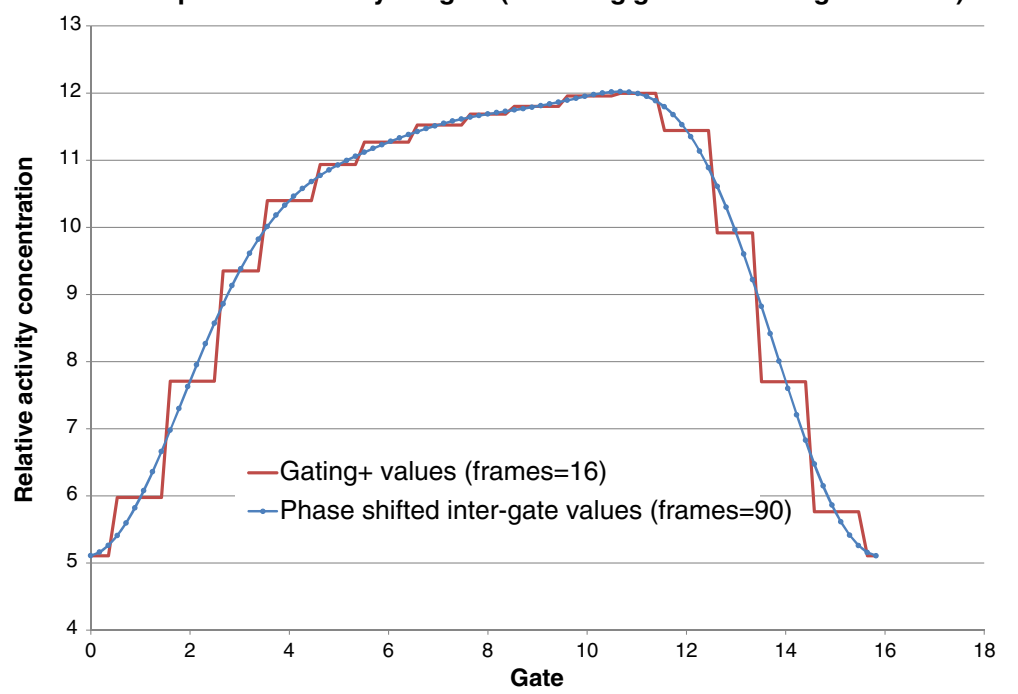

Figure 2 Example voxel activity curve. Taken from rat FDG $\mu$ PET image (16 gating + gates, one respiratory cycle). Gating+ (gated) values presented with corresponding phase-shifted inter-gate values. Image illustrates a high correlation of gate values with inter-gate values.

manually placed to characterize the lung/liver boundary. Background noise levels were assessed in all 84 datasets by manually placing a 0.2 -cc (approximately 400 voxels) spherical volume of interest in low-uptake areas of the shoulder, or the hip region in cases where no shoulder was available.

\section{Results}

\section{Software and hardware comparison}

The 24 scans that had been constructed using both hardware- and software-based respiratory triggers were assessed and compared (Table 1). The median respiratory periods measured from hardware and software triggers were within $5 \%$ of each other for $22 / 24$ scans, $\pm 5 \%$ being the envelope that can be expected due to the difference in timing resolutions: software at $100 \mathrm{~ms}$ and hardware at $10 \mathrm{~ms}$.

\section{Software gating}

All 84 scans were processed to yield 4D gated images. Figure 3 shows an example of a software-gated scan. The majority of software-gated images, 63/84 (75\%), were confirmed to have characteristic respiratory motion as assessed by four independent reviewers.

The maximum COM displacement values were $0.71 \pm$ $0.46 \mathrm{~mm}$ (0.16 to $1.89 \mathrm{~mm}$ ) (mean $\pm \mathrm{SD}$ (range)) for the software-gated images. A summary of the COM data and its correlation with observer data is illustrated in Figure 4. The maximum COM data for the corresponding randomly gated scans are also shown in this figure. We observed a correlation between count statistics and random COM displacements. An envelope - defined as the moving average of the random measurements plus two standard deviations (also shown in Figure 4) - was subsequently used to characterize a count-specific threshold that delineates significant/nonsignificant displacements resulting from gating. Those software gating COM measurements lying above this cutoff ( $n=60 / 84,71 \%)$ were considered to reflect respiratory motion identified by gating. The software gating COM measurements lying below this threshold were considered to possibly be an expression of noise only. This cutoff is approximate and based on a statistical probability that approximately $97.8 \%$ of random measurements will fall below this cutoff, and is used to provide a quantitative separation between verified true respiratory motion and noise. While scans with low COM results may indicate incorrectly gated data, it is also possible that these are correctly gated scans that would not benefit from gating, an idea supported by our hardware-software comparison.

Scans with lower total counts tended to have lower COM displacement values, but there was no global correlation (Pearson correlation coefficient $=0.13$ ). Scans that were qualitatively assessed by reviewers to exhibit motion had on average $2.6 \times 10^{8}$ true counts, while non-motion scans had on average $59 \%$ less. Scans that had observable uptake in the heart and/or liver had a higher rate of reviewer-confirmed motion, 82\% (49/60). Scans that did not have uptake in the heart nor liver, including scans that did not include this region in the FOV, had a lower rate of confirmed motion 58\% (14/24).

\section{Gating+ gate recombination}

Gated volumes were processed to create gating+ volumes both for the small-animal PET scans and simulations. Gating+ images consistently exhibited resolution improvement similar 
Table 1 Hardware-vs.-software comparison

\begin{tabular}{|c|c|c|c|c|c|c|c|c|}
\hline \multirow{2}{*}{$\begin{array}{l}\text { Scan } \\
\text { number }\end{array}$} & \multirow{2}{*}{$\begin{array}{l}\text { Events in } \\
\text { scan (trues), } \times 10^{8}\end{array}$} & \multicolumn{3}{|c|}{ Median respiratory period (s) } & \multicolumn{3}{|c|}{ Maximum COM displacement (mm) } & \multirow{2}{*}{$\begin{array}{l}\text { Motion observed } \\
\text { by all four } \\
\text { independent observers }\end{array}$} \\
\hline & & Software & Hardware & Difference & Software & Hardware & Difference & \\
\hline 1 & 1.4 & 1.00 & 0.98 & 0.02 & 1.16 & 1.20 & -0.04 & Yes \\
\hline 2 & 1.4 & 1.20 & 1.23 & -0.03 & 0.47 & 0.33 & 0.14 & Yes \\
\hline 3 & 1.6 & 1.10 & 1.06 & 0.04 & 0.96 & 0.91 & 0.05 & Yes \\
\hline 4 & 1.6 & 1.20 & 1.21 & -0.01 & 1.37 & 1.89 & -0.52 & Yes \\
\hline 5 & 1.6 & 1.30 & 1.26 & 0.04 & 0.28 & 0.37 & -0.09 & Yes \\
\hline 6 & 1.9 & 1.10 & 1.07 & 0.03 & 1.48 & 1.40 & 0.08 & Yes \\
\hline 7 & 2.1 & 1.20 & 1.22 & -0.02 & 0.19 & 0.21 & -0.02 & Yes \\
\hline 8 & 2.1 & 1.20 & 1.15 & 0.05 & 1.43 & 1.51 & -0.08 & Yes \\
\hline 9 & 2.3 & 1.10 & 1.12 & -0.02 & 0.94 & 0.92 & 0.02 & Yes \\
\hline 10 & 2.5 & 0.90 & 1.18 & -0.28 & 0.26 & 0.16 & 0.10 & No \\
\hline 11 & 2.6 & 1.20 & 1.23 & -0.03 & 1.38 & 1.55 & -0.17 & Yes \\
\hline 12 & 2.6 & 1.40 & 1.35 & 0.04 & 0.21 & 0.20 & 0.01 & Yes \\
\hline 13 & 2.7 & 1.20 & 1.24 & -0.04 & 0.58 & 0.58 & -0.01 & Yes \\
\hline 14 & 2.9 & 1.20 & 1.15 & 0.05 & 1.08 & 1.03 & 0.04 & Yes \\
\hline 15 & 2.9 & 1.10 & 1.06 & 0.04 & 0.34 & 0.32 & 0.02 & Yes \\
\hline 16 & 3.0 & 1.20 & 1.22 & -0.02 & 0.26 & 0.33 & -0.07 & Yes \\
\hline 17 & 3.1 & 1.20 & 1.30 & -0.10 & 0.21 & 0.26 & -0.05 & No \\
\hline 18 & 3.1 & 1.20 & 1.17 & 0.03 & 0.35 & 0.37 & -0.03 & Yes \\
\hline 19 & 3.5 & 1.10 & 1.14 & -0.04 & 0.19 & 0.23 & -0.04 & Yes \\
\hline 20 & 3.5 & 1.20 & 1.16 & 0.04 & 0.16 & 0.19 & -0.03 & Yes \\
\hline 21 & 3.9 & 1.10 & 1.06 & 0.04 & 1.36 & 1.27 & 0.09 & Yes \\
\hline 22 & 4.2 & 1.20 & 1.16 & 0.04 & 1.49 & 1.51 & -0.02 & Yes \\
\hline 23 & 4.8 & 1.10 & 1.07 & 0.03 & 1.30 & 1.47 & -0.17 & Yes \\
\hline 24 & 4.9 & 1.10 & 1.10 & 0.00 & 1.07 & 1.12 & -0.06 & Yes \\
\hline
\end{tabular}

Summary of median respiratory periods and maximum COM displacement for 24 scans acquired using both software and hardware gating triggers.

to that of the gated scans while maintaining lower noise levels in uniform or low-count areas. Simulations with a moving lesion are shown in Figure 5, and corresponding measurements from that data are displayed in Table 2, confirming favorable resolution and noise measurements in the gating+-processed images. Also seen in Figure 5 are the motion maps created as part of the gating+ processing.

To assess the gating+ accuracy, we generated 2,000 realizations of the moving lesion for each noise condition. There was a probability $(P>0.5)$ that the gating+ value was more accurate than the ungated value in every voxel in the simulations. We also found a probability $(P>0.5)$ of the gating+ value being more accurate than the gated value in $98 \%$ of the voxels. This last $2 \%$ occurred in voxels that had very high statistics, possibly because highest frequencies were not preserved in the gating+ method.

To put the gating+ algorithm in perspective relative to other temporal filtering techniques, our simulation scenario was also processed using ramp and Wiener filters applied in the time domain, with results shown in Figure 6 . The Wiener filtering approach we used is described by King and Miller [28].

In the preclinical PET scans, the maximum COM displacement for the population was $0.43 \pm 0.41 \mathrm{~mm}$ (0.04 to $1.47 \mathrm{~mm})$ (mean $\pm \mathrm{SD}$ (range)) for the gating+ images. Kidney VOIs were definable for 52 scans, and liver profiles, 34 scans. A summary of the quantitative measurements is presented in Table 3. Shoulder/hip background VOIs were defined in all 84 scans. The average relative percent SDs in background regions for the gated, gating+, and ungated images were 1.51, 1.05, and 1.00 , respectively, indicating superior noise presentation in ungated and gating+ images. All gating and gating+ measurements shown in Table 3 exhibited statistically significant effects (paired $t$ test $P<0.01$ ), indicating that gating + had a measureable effect for all measurements. The filtering of high frequencies during the gating+ processing did not change the total activity value (summed over all gates) for any voxel - activity was conserved. The potential for introducing negative activity values does 


\section{Example of software based gated scan. Average Intensity Projection Images}

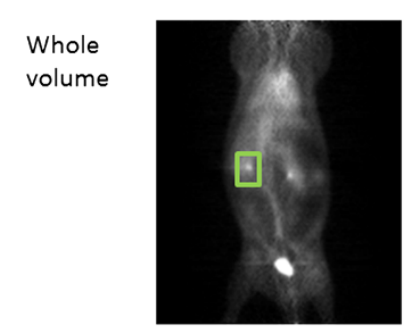

Region of interest (gated)

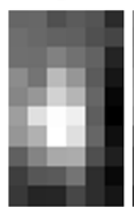

Gate 1

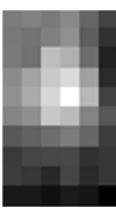

Gate 2
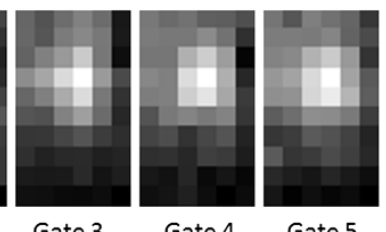

Gate 5

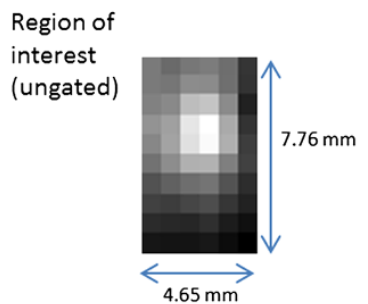

Figure 3 Example software-gated image. Projection image illustrating motion of kidney seen using software-based gating methods. Image data were acquired using a standard (ungated) acquisition.

exist, but only in rare instances where the low-frequency fluctuations have a greater magnitude than the mean (DC) voxel value, and in these cases, it is only at minimal amounts. In the population of small-animal PET scans, $0.09 \%$ of voxels had negative values, and they were almost exclusively located in areas with no true activity, with a mean absolute negative activity of approximately $10^{-10}$ of the total scan activity.

An example small-animal PET image showing the noise differences between gated and gating+ images is shown in

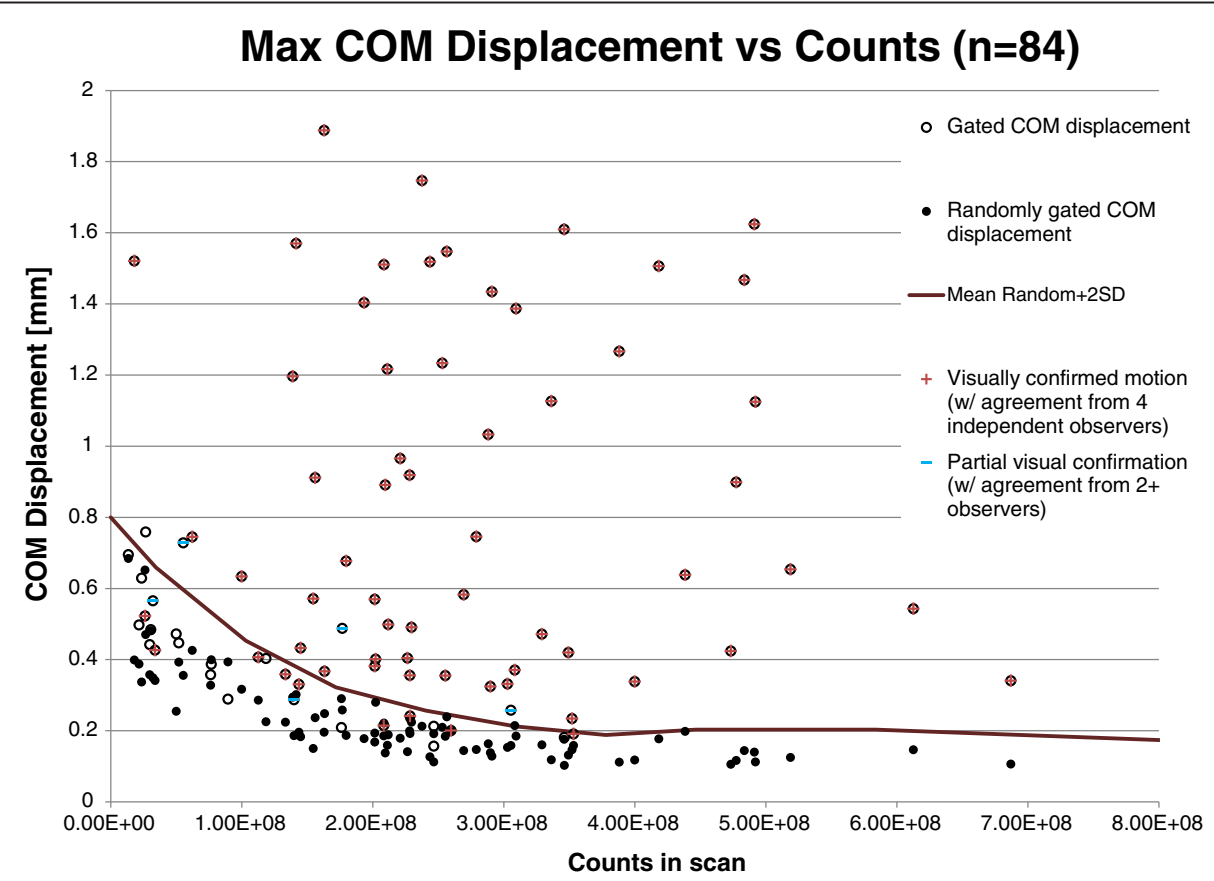

Figure 4 Center of mass displacements in respiratory gated images. Maximum center of mass displacements are displayed for the population of small-animal PET scans for both respiratory motion gated and randomly gated datasets. The moving average of the values corresponding to the randomly gated data plus two standard deviations is also displayed. This threshold was used to characterize the scans (those lying above the line) that were enhanced with motion as a result of software gating. 


\section{$\underline{\text { Relative count statistics }}$}

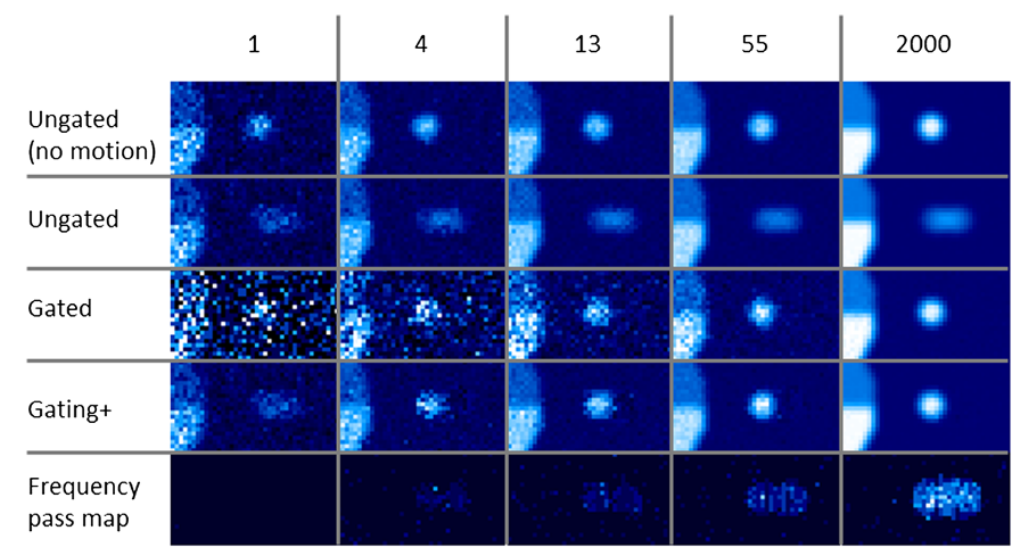

Figure 5 Lesion motion simulations. Gated simulation of a hot lesion moving in sinusoidal motion (left to right) over a colder background next to a stationary boundary. Simulated with 16 gates. The effective views show (top to bottom) count-equivalent ungated images with no motion, ungated images with motion, and a single-gate gated image, a corresponding gating+ image and a respective motion maps generated during the gating+ processing. Differing scan conditions are shown with lower count scenarios on the left, ranging to higher count scenarios towards the right. Lesion displacement $=60 \%$ lesion diameter. Lesion/background ratio $=3$. Upper diaphragm/background $=1.5$. Lower diaphragm/background = 3.0. Linear color scale.

Figure 7. Resolution and motion effects for the rat shown in Figure 7 (example A) are illustrated with a sample line profile in Figure 8. It is seen that gated and gating+ images exhibit concentrations of activity at different locations in the different gates, an expected consequence of motion.

Table 2 Simulation measurements

\begin{tabular}{|c|c|c|c|c|c|}
\hline Relative counts in image space & 1 & 4 & 13 & 55 & 2,000 \\
\hline \multicolumn{6}{|l|}{ Maximum } \\
\hline Ungated & 94 & 82 & 78 & 75 & 74 \\
\hline Gated & 219 & 146 & 122 & 108 & 100 \\
\hline Gating+ & 95 & 125 & 115 & 108 & 100 \\
\hline \multicolumn{6}{|l|}{ Volume (70\% maximum) } \\
\hline Ungated & 57 & 144 & 177 & 194 & 199 \\
\hline Gated & 6 & 17 & 49 & 84 & 99 \\
\hline Gating+ & 55 & 41 & 76 & 89 & 103 \\
\hline \multicolumn{6}{|l|}{ SUV (mean VOI/background) } \\
\hline Ungated & 91 & 77 & 74 & 73 & 72 \\
\hline$\overline{\text { Gated }}$ & 249 & 153 & 118 & 104 & 100 \\
\hline Gating+ & 92 & 122 & 110 & 105 & 100 \\
\hline \multicolumn{6}{|l|}{ FWHM } \\
\hline Ungated & 144 & 174 & 183 & 187 & 188 \\
\hline Gated & 38 & 68 & 90 & 98 & 100 \\
\hline Gating+ & 143 & 88 & 96 & 94 & 100 \\
\hline \multicolumn{6}{|c|}{$\begin{array}{l}\text { Summary of lesion measurements from simulations of hot lesion against } \\
\text { colder background with a range of random noise levels added (see Figure 5). } \\
\text { Numbers represent average measurements from a single gate of } 500 \\
\text { simulations for each noise scenario. All measurements are presented as a } \\
\text { percentage of the corresponding true noise-free values. The standardized } \\
\text { uptake value (SUV) was calculated from for } 70 \% \text { threshold volume. }\end{array}$} \\
\hline
\end{tabular}

The gated profiles have a slimmer width indicating improved image resolution relative to the ungated image. The change in the magnitude of activity concentration is an indication that activity is moving in three dimensions, traversing the coronal image plane. An image sequence, highlighting both noise and resolution/motion benefits resulting from processing, can be seen in Additional file 1.

\section{Phase-shifted CMI images}

Phase-shifted images were generated to model continuous motion, with 90 frames/cycle. Figure 9 shows example liver boundary location measurements as determined from respective gates and CMIs. Table 3 shows that uptake measurements from these images were very similar to the gating+ images - kidney uptake measurements, liver displacement measurements, and background noise

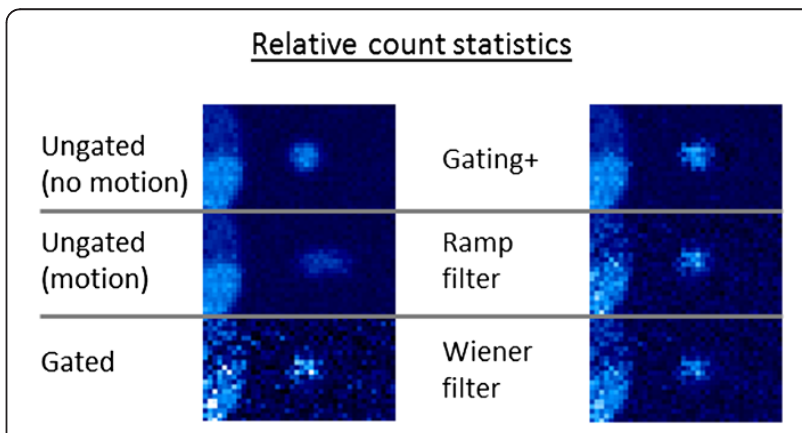

Figure 6 Comparison of temporal filtering techniques using lesion simulation. All filters are applied solely in temporal space. 
Table 3 Preclinical PET motion effect measurements

\begin{tabular}{|c|c|c|c|c|c|c|c|}
\hline & & & \multirow[t]{2}{*}{ Units } & \multicolumn{4}{|c|}{ Measurements } \\
\hline & & & & Gated & Gating+ & $\begin{array}{l}\text { Gating+ with } \\
\text { phase shift }\end{array}$ & Ungated \\
\hline $\begin{array}{l}\text { Number of frames } \\
\text { (phase increment }=360^{\circ} / \text { number) }\end{array}$ & & & & 16 & 16 & 90 & 1 \\
\hline \multirow[t]{3}{*}{ Kidney VOI, $n=102$} & $\begin{array}{l}\text { Average kidney } \\
\text { uptake }\end{array}$ & $\begin{array}{l}\text { Average } \mathrm{VOI} \text { value, average } \\
\text { of all gates }\end{array}$ & Relative values & 1.01 & 1.01 & 1.01 & 1.00 \\
\hline & $\% \mathrm{SD}$ & Average uptake across gates & Percentage & 2.69 & 1.47 & 1.47 & \\
\hline & $\begin{array}{l}\text { Maximum } \\
\text { displacement }\end{array}$ & $\begin{array}{l}\text { Average of all scans } \\
\text { (SD of all scans) }\end{array}$ & $\mathrm{mm}$ & $\begin{array}{l}1.50 \\
(0.56)\end{array}$ & $1.10(0.75)$ & $1.10(0.75)$ & \\
\hline Liver profile, $n=34$ & $\begin{array}{l}\text { Liver boundary } \\
\text { displacement }\end{array}$ & & $\mathrm{mm}$ & 3.25 & 3.04 & 3.05 & \\
\hline Shoulder VOI, $n=84$ & $\% \mathrm{SD}$ in $\mathrm{VOI}$ & Average of all gates & Relative values & 1.53 & 1.05 & 1.05 & 1.00 \\
\hline Global COM, $n=84$ & $\begin{array}{l}\text { Maximum COM } \\
\text { displacement }\end{array}$ & $\begin{array}{l}\text { Average of all scans } \\
\text { (SD of all scans) }\end{array}$ & $\mathrm{mm}$ & $\begin{array}{l}0.71 \\
(0.46)\end{array}$ & $0.43(0.41)$ & $0.43(0.41)$ & \\
\hline
\end{tabular}

Summary of VOI, line profile, and COM measurements in ungated, gated, and gating+ images across the population of preclinical PET scans. All numbers are average values of population or all appropriate population scans.

measurements averaged over the population were all within $1 \%$ of each other for the gating+ images and CMIs. Samples of the continuous motion image sequences can be seen in Additional files 2 and 3. A side-by-side comparison of corresponding gated and gating+ embodiments of selected scans with different levels of useful motion information is shown in Additional file 4, illustrating how the algorithm passes useful information while filtering noise.

To gain insight into the accuracy of the CMI measurements, simulations were constructed using randomly generated time-activity curves. Voxel activity vs. phase curves were generated randomly with signals in frequencies <Nyquist frequency. Gated (step function) values were derived from the true curves; CMI values were derived from gated values and evaluated. In $100 \%$ of the simulations $\left(10^{6}\right)$, the CMI curves correlated better with true motion curves than the respective gated curves.

The time required to process a 10-min acquisition from listmode, generating ungated, gated, and gating+ images, was approximately $1.5 \mathrm{~h}$ using a standard PC. Because the software we used in this study is proprietary, we were not

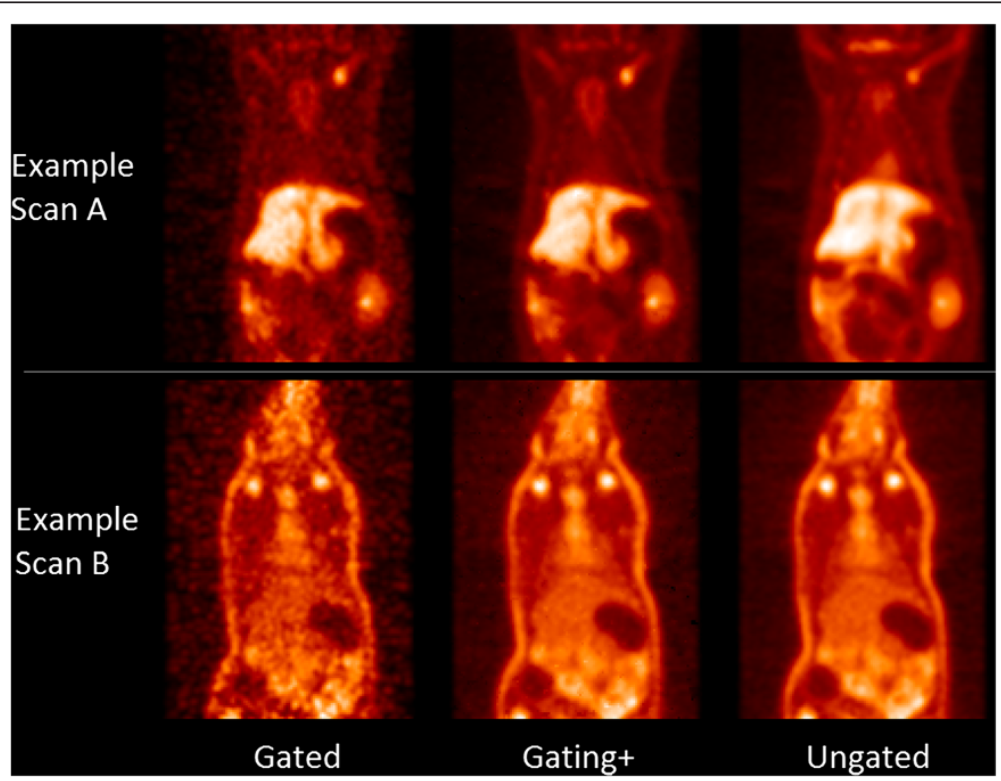

Figure 7 PET images (coronal slice) illustrating differences in signal quality between gated, gating+, and ungated images. In example A, the activity in the ribs is not well discernible from its surroundings in the gated image; however, in the corresponding gating+ image, the bones have discernible contrast. The gating+ image also shows better definition of the liver and kidneys. In example B, we again see discernible boundaries of the liver and ribs in the gating+ image and not in the gated image. Both images also highlight the global noise reduction provided by gating+. Example A was acquired with ${ }^{18} \mathrm{~F}-\mathrm{FDG}$, and example $\mathrm{B}$ was acquired with ${ }^{18} \mathrm{~F}-\mathrm{FBDPMA}$. 


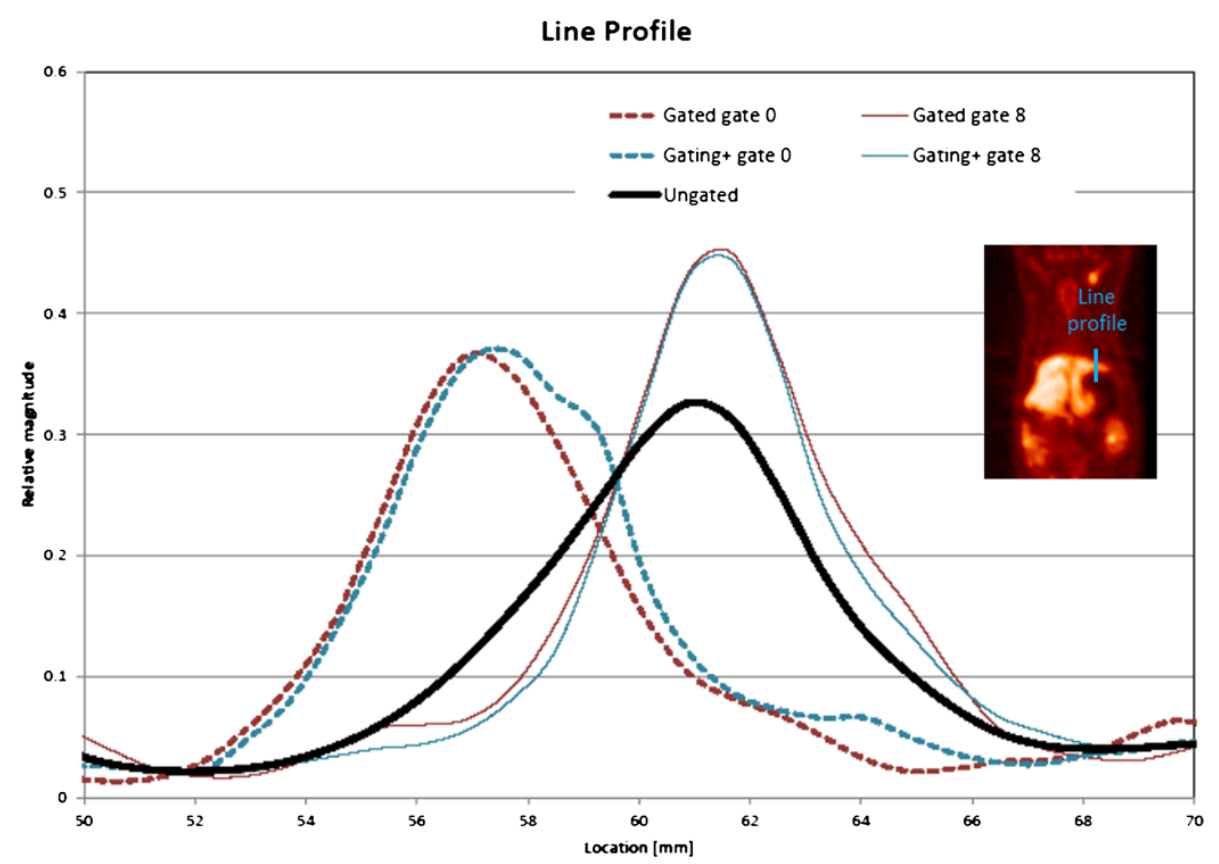

Figure 8 Example motion profile. Illustration of line profile placed on the rat shown in Figure 7, example A. Gated, gating+, and ungated profiles illustrate motion and spatial resolution characteristics.

able to optimize this processing. However, in our previous work [18], we showed that this processing time can be drastically reduced, to approximately the time of scan acquisition or better, if integrated with vendor software. The gating+ algorithm required approximately $20 \mathrm{~s}$ of processing once the requisite source images were available. Phase-shifted images required approximately $0.02 \mathrm{~s} / 2 \mathrm{D}$ image slice to generate.

\section{Discussion}

In this study, we have shown that data-driven gating methods previously demonstrated in human PET can be extended to small-animal PET and used with a large scan population, with diverse radiotracers and activity distributions. Furthermore, to generate less noisy gated images, we developed an algorithm based on voxel-specific temporal filtering to yield optimized gated images, denoted as gating+. The two techniques can be used separately or combined together into a motion correction workflow as we have done here. Both algorithms utilize motion information that is inherently contained in PET data and use it to create optimized images.

Data-driven and hardware-based gating are two very different approaches for acquiring essentially the same respiratory signature for a scan. Identical results cannot be expected from hardware and data-driven gating since hardware gating is based on signal recorded by a small device (in our case, an 18-mm-diameter pressure-sensitive pad placed under some part of the thorax of the rat), while the data-driven algorithm used in this study combines respiratory motion signals identified as periodic changes in activity concentration at any and all locations in the image volume. Nevertheless, in this work, visual and quantitative assessments confirmed that the two methods performed similarly with respect to trigger points and 4D image presentation for a subset of 24 scans, as has been observed by others $[12,15]$. In this assessment, however, we can note that there were two $(/ 24)$ scans in which the median measured periods for these scans differed between hardware and software signal, and for which observers did find motion. Closer inspection revealed that for the first scan, both the hardware and software respiratory traces appeared very erratic, with significant portions of the scan interval exhibiting frequent large changes $(>20 \%)$ in periodicity. Such behavior precludes useful respiratory gating, irrespective of the gating system. For the second scan, hardware gating yielded observable respiratory motion on the reconstructed images, while the software failed. Further scrutiny revealed that modifications to the frequency window parameter used in the software gating process could fix the error. This example highlights the importance of optimizing gating parameters and is discussed more below.

For the entire series of 84 scans, as expected, varying magnitudes of motion were observed in the gated and gating+ images, presumably reflecting different sizes of the rats and different levels of respiration. Our scan population also included some scans where the thorax and abdomen were almost entirely outside the axial FOV, and it 
was clearly appropriate that minimal, if any, respiratory motion should be detected in these scans. In such cases, there is no additional information to be gained from gating, yet there will be a cost in subdividing statistics. The combination of data-driven gating with gating + signal optimization ensures that in such a case of inappropriate gating, the gating+ image will essentially default to its ungated state. This behavior is exemplified in Additional file 4.

For most PET scans, at least some respiratory information was captured - $75 \%$ of the scan population had motion confirmed by independent reviewers, and 71\%, quantitatively using a global COM measurement. Liver profiles and kidney VOIs also identified characteristics of respiratory motion. On the other hand, we did not see significant change in SUV in the kidneys between gated and ungated scans, which may have been expected from analogous studies assessing lung lesions [21,34]. We suspect that kidney motion and uptake were not sufficient to show this effect.

In addition to extending software gating methods to preclinical PET, this work also addressed a fundamental consequence of gating: that gated images include less motion, but also fewer counts. This inherent trade-off presents a dilemma which inhibits the robust utilization of gating and provided the motivation to develop methods to improve the noise introduced in gated images.

The gating + algorithm we presented is based on the filtering of signal in the temporal domain. In Figure 6, we can see a scenario where gated data were reconstructed using several filters: gating+, ramp, and Wiener. It is seen in this example that the gating+ algorithm is flexible enough to recover lesion resolution not present in the ungated image while also preserving background contrast not present in the gated image, and it handles this spectrum better than other filters, which provide more uniform filtering effects across the image. The other family of strategies used for addressing the poor statistics resulting from gating is based upon nonlinear motion mapping [22-24]. In an initial attempt to apply optical flow algorithms [22] to improve our gated images, the techniques appeared to work well for some scans yet performed prohibitively badly for others. One of the difficulties, common to these as well as other nonlinear image-morphing algorithms, lies in the need for calibrating definitions of signal and noise and in determining the freedom allowed in the mapping process. These parameters need to provide enough flexibility to achieve accurate results while avoiding the hazard of generating (potentially good-looking) images that are inaccurate. In diverse populations like that presented in this study, which include different noise levels, biodistributions, and FOVs, finding optimal algorithm parameters can prove difficult.

In contrast to motion mapping, the algorithm we are presenting is designed to avoid such problems, and all the available signals are optimized using a single onedimensional temporal frequency filtering equation applied to the data for each voxel of the image volume. Scanspecific factors - complex activity distributions, poor scan conditions, variable noise levels - are all assessed and handled the same way. Since the algorithm is not dependent

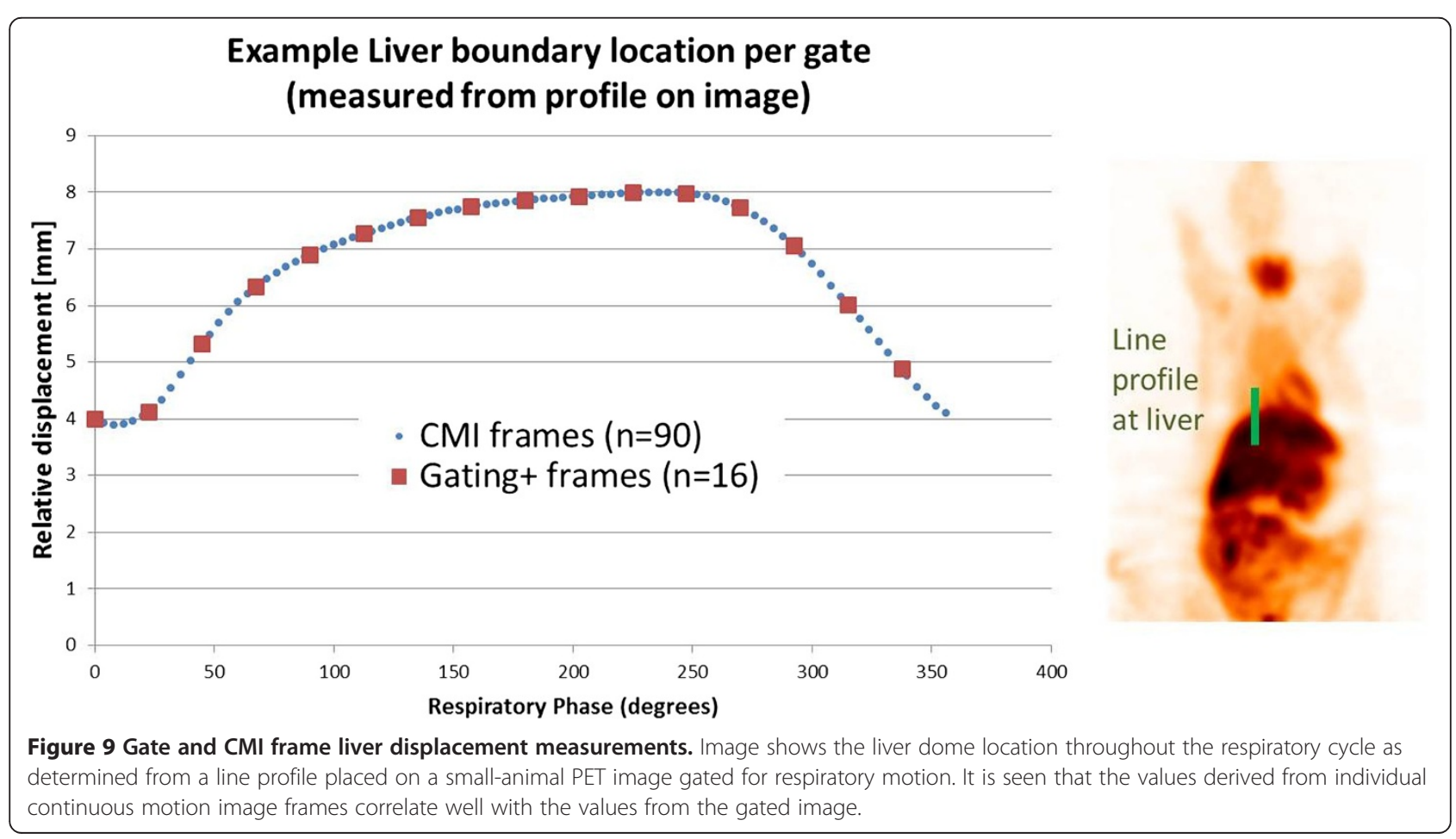


on scan conditions, it is characterizable, reproducible, and automated. Our software for creating gating+ images is built of about 15 lines of high-level (IDL) code, requires only seconds of processing per PET volume, and produces as output complete 4D image sets with associated frequency pass maps. Potential errors in gating+ images are limited by the fact that they are created with selective use of raw information. The fact that no higher frequencies are passed without supporting lower frequencies avoids the danger of unpredictable jumps in signal or Gibbs artifacts.

The implementation of the gating+ algorithm involves assessment of signal at every voxel in every frequency, selectively including the fluctuating signal due to respiratory motion only when and where it is not confounded by noise. To characterize the difference between useful signal and noise in a per-voxel perfrequency basis, we used an estimated threshold of $1.2 \times$ effective noise. The effective noise is derived from the randomly gated image. The constant 1.2 was derived from Monte Carlo simulations: $10^{8}$ combinations of ranging magnitude and phase scenarios for motion and noise vectors were simulated. Both motion and noise vectors contain an element of noise which comes with random phase. We modeled this random process and found that when the ratio between motion and noise vector magnitudes is greater than approximately 1.2 , then it becomes more probable $(P>0.5)$ that the gated signal is closer to the true signal than the ungated signal. In essence, the unknown phase of the noise is managed through knowledge of its magnitude, random phase, and statistical behavior, which allows us to make a binary determination as to its likely benefit on the accuracy of the gated signal. The concept may be understood as such: intuitively, where the true motion signal vectors are much greater in magnitude than the noise vectors, the gated signal is more reliable regardless of the noise and should be used. When the signal-to-noise ratio is poor, then useful fluctuations will be indiscernible through the noise, thus the gated signal provides no added value and should not be used. The implication of this strategy is that a gating+ voxel value will, on average, have improved accuracy relative to its ungated value.

When implementing gating, there is an important question of precision and accuracy of the motion capture. All forms of data-driven gating algorithms have several parameters which should be optimized to get the most favorable results: frequency pass windows, time bin duration, reconstruction parameters, etc. Then, the gating process too has parameters to be considered as well: trigger definition, data bin formation, number of histograms, etc. The significance of these issues were made clear to us when we found that changes in the frequency pass windows, used in the data-driven gating process, could affect the final results. However, changing the window to accommodate one scan degraded the quality of another, making it difficult to optimize the parameters.

Understanding this issue of what constitutes an optimal and non-optimal signal is of great importance as the field of gating moves forward in both the software-based and hardware-based arenas. Patient motion, uptake patterns, and scan statistics are very case specific. With hardware gating, results are variable with respect to the placement of monitoring devices and particular patient geometry/ behavior, and parameter optimization in software gating can be understood as analogous. Our work demonstrated to us, however, that there may be a large advantage with software gating in that scans can be reprocessed retrospectively in an effort to achieve an optimal signal. There is potential for future methodological advancements in data-driven gating to incorporate iterative steps that will optimize all parameters during processing, making the algorithm more appropriate for use in diverse populations, possibly using the concept of a motion score [19]. Datadriven gating research could expand the classic concept of motion control achieved through gating towards algorithms that extract an optimal motion signal and present it with an optimal benefit.

While implementing the gating+ algorithm, we noticed some limitations/behaviors of the processing that we hope to address in future development of the algorithm. In voxels which have selective frequencies filtered, there is the potential to have a 'shadow effect' resulting from the fact that the true curve cannot be sufficiently modeled using the available lower-frequency sinusoidal waveforms passed in a band-pass filter. This effect can be seen in our simulations (Figure 2) where regions in the path of motion appear slightly darker than the background. We could not, however, find this effect in our preclinical images, likely because actual images have non-ideal statistical properties and deviate from perfect sinusoidal motion. However, because the gating+ voxel values are defined by the 'optimal' frequencies, they are still more likely to be accurate than the ungated values even if they are affected by this shadow. Future work can explore correcting this issue probably through a strategy of partial filtering in some frequencies, such as combining a Wiener filtration strategy with our approach for noise estimation, as opposed to the allor-nothing band-pass approach we used here.

In addition to the images, the gating+ process creates a frequency pass 'motion map' that describes the distribution of motion information (Figure 5, Additional file 1). In future work, this map can potentially be used for motion characterization, lesion detection, gating optimization, or other gate utilization algorithms.

Also, we have begun to explore the ability for generating CMIs from 4D data. We are not creating any information to generate additional frames; rather, we are managing 
information that is available in a more flexible manner: we are considering gated data to define a step function in frequency space rather than in image space. While the gating+ processing is not a requirement for creating CMIs, the approach of optimizing signal in frequency space readily prepares the signal to be visualized at a user-defined phase while maintaining optimal statistics. The combined processes, illustrated in Figures 2 and 9, may offer intuitive presentations of motion, a new platform for understanding patterns in patient motion and organ-phase relationships, provide sub-gate activity derivatives which may be used for enhancing optical flow and/or other nonlinear mapping processes, and possibly present a new paradigm for understanding the trade-off between the signal/noise ratio vs. number of gates.

Motion control in nuclear medicine imaging currently remains a major obstacle impeding further resolution advancements. Despite a plethora of options to help address respiratory motion correction in PET, no clear optimal approach has emerged. Current commercial options for respiratory gating all use hardware which requires extra cost, time, effort, and training. Current gating research requires subjecting patients to additional scans [5]. In this work, we aimed to demonstrate that exclusively datadriven methodology for gating in PET is entering a new stage where software-based algorithms can create motioncorrected/noise-filtered scans in a fast and fully automated manner. Our methods use information that is present in the data and is not currently utilized. Signal optimization strategies like the one we are presenting provide a practical alternative for motion control in PET and may turn the long acquisition times required in nuclear medicine, traditionally considered a drawback, into a benefit.

\section{Conclusions}

Data-driven gating and gating+ for image enhancement offer a strategy for creating motion-corrected images from ungated acquisitions that have noise characteristics similar to ungated images. The methodology was demonstrated on preclinical PET images with diverse activity distributions but should be equally applicable to clinical PET or other modalities. Future work will focus on improving methods and documenting clinical benefits of motion control. Data-driven gating and gating+ methods may be expanded to handle cardiac and other types of motion as well as other modalities, including SPECT/gamma camera imaging, CT, and ultrasound.

\section{Additional files}

Additional file 1: Figure S1. Example of a coronal slice from a smallanimal PET rat scan. From left to right: summed (i.e., ungated) image, the same image, gated using software-based gating, same image with 'gating+' processing. On the right: motion map generated by the gating + algorithm, where a higher signal indicates a higher cutoff frequency. Color scale is shown to the right of the motion map. Scan was reconstructed with 16 gates.

Additional file 2: Figure S2. Example of an image slice. On the left, the image is shown in its gated embodiment. On the right, the same image is corrected using gating + and shifted in phase to generate 90 frames distributed between 0 to $360^{\circ}$.

Additional file 3: Figure S3. Examples of maximum-intensity projection images created using automated software gating, gating + signal combination, and phase offset (30 frames/s) processes. Images displayed rotating $360^{\circ}$ through cycle.

Additional file 4: Figure S4. Central slices of eight small-animal PET scans are displayed in gated and gating+ form. Top four sets illustrate the gated and gating + form of the scans which contain useful motion information. It is seen that the gating + images maintain motion information while suppressing noise. The bottom four sets illustrate scans that do not benefit from gating. In these scans, we see that the reduction of image quality caused by gating is minimized in the gating+ images.

\section{Competing interests}

Methods described here have been submitted for a US provisional patent.

\section{Authors' contributions}

ALK devised the project, developed the methods, helped with the image acquisition, processed the data, and drafted the manuscript. GA and EM were involved in the image acquisition, project design, and drafting of the manuscript. RS and ST were involved in drafting of the manuscript. NF acted as senior PI and aided with the project design, data acquisition, and drafting of the manuscript. All authors read and approved the final manuscript.

\section{Acknowledgements}

We wish to acknowledge the Fulbright commission for Israel and the United States-Israel Educational Foundation which provided partial funding and support for this project. We would also like to thank Aposense for kindly sharing their imaging data. We thank Iska Paniri for assisting as a motion assessment reviewer.

\section{Author details}

${ }^{1}$ Department of Medical Biophysics and Nuclear Medicine, Kiryat Hadassah, P.O. Box 12000, Jerusalem 91120, Israel. ${ }^{2}$ Cyclotron/Radiochemistry Unit, Hadassah University Hospital, Kiryat Hadassah, P.O. Box 12000, Jerusalem 91120, Israel.

Received: 9 December 2012 Accepted: 5 March 2013 Published: 24 April 2013

\section{References}

1. Baum SH, Frühwald M, Rahbar K, Wessling J, Schober O, Weckesser M: Contribution of PET/CT to prediction of outcome in children and young adults with rhabdomyosarcoma. J Nucl Med 2011, 52:1535-1540. doi:110.082511.

2. Groheux D, Giacchetti S, Espié M, Vercellino L, Hamy AS, Delord M, Berenger $\mathrm{N}$, Toubert ME, Misset JL, Hindié E: The yield of 18F-FDG PET/CT in patients with clinical stage IIA, IIB, or IIIA breast cancer: a prospective study. J Nucl Med 2011, 52:1526-1534. doi:111.093864.

3. Daou D: Respiratory motion handling is mandatory to accomplish the high-resolution PET destiny. Eur J NuCl Med Mol Imaging 2008, 35:1961-1970. doi:10.1007/s00259-008-0931-x.

4. Bettinardi V, Picchio M, Di Muzio N, Gianolli L, Gilardi MC, Messa C: Detection and compensation of organ/lesion motion using 4D-PET/CT respiratory gated acquisition techniques. Radiother Oncol 2010, 96:311-316. doi:S0167-8140(10)00421-4.

5. Guerra L, De Ponti E, Elisei F, Bettinardi V, Landoni C, Picchio M, Gilardi MC, Versari A, Fioroni F, Dziuk M, Koza M, Ahond-Vionnet R, Collin B, Messa C: Respiratory gated PET/CT in a European multicentre retrospective study: added diagnostic value in detection and characterization of lung lesions. Eur J Nucl Med Mol Imaging 2012, 39:1381-1390. doi:10.1007/s00259-012-2148-2. 
6. Schäfers KP, Lang N, Stegger L, Schober O, Schäfers M: Gated listmode acquisition with the quadHIDAC animal PET to image mouse hearts. ZMed Phys 2006, 16:60-66.

7. Boucher L, Rodrigue $S$, Lecomte R, Bénard F: Respiratory gating for 3-dimensional PET of the thorax: feasibility and initial results. J NuCl Med 2004, 45:214-219.

8. Nehmeh SA, Erdi YE, Ling CC, Rosenzweig KE, Squire OD, Braban LE, Ford E, Sidhu K, Mageras GS, Larson SM, Humm JL: Effect of respiratory gating on reducing lung motion artifacts in PET imaging of lung cancer. Med Phys 2002, 29:366-371.

9. Bundschuh RA, Martinez-Moller A, Essler M, Nekolla SG, Ziegler SI, Schwaiger $\mathrm{M}$ : Local motion correction for lung tumours in PET/CT-first results. Eur J Nucl Med Mol Imaging 2008, 35:1981-1988.

10. Detorie NC, Kesner AL, Solberg TD, Dahlbom M: Evaluation of image noise in respiratory gated PET. IEEE Trans NUCI SCi 2007, 54:66-70.

11. van Elmpt W, Hamill J, Jones J, De Ruysscher D, Lambin P, Ollers M: Optimal gating compared to 3D and 4D PET reconstruction for characterization of lung tumours. Eur J Nucl Med Mol Imaging 2011, 38:843-855. doi:10.1007/s00259-010-1716-6.

12. Schleyer PJ, O'Doherty MJ, Marsden PK: Extension of a data-driven gating technique to 3D, whole body PET studies. Phys Med Biol 2011, 56:3953-3965. doi:S0031-9155(11)74643-7.

13. Mostov K, Liptsen E, Boutchko R: Medical applications of shortwave FM radar: remote monitoring of cardiac and respiratory motion. Med Phys 2010, 37:1332-1338.

14. Woo S-K, Song TY, Choi JY, Hong KJ, Choi Y, Lee K-H, Kim BT: Development of a motion detecting system for respiratory-gated PET using laser optical displacement sensor. In Nuclear Science Symposium Conference Record: 2003 (19-25 Oct. 2003). Portland: IEEE.

15. Büther F, Ernst I, Dawood M, Kraxner P, Schäfers M, Schober O, Schäfers KP. Detection of respiratory tumour motion using intrinsic list mode-driven gating in positron emission tomography. Eur J Nucl Med Mol Imaging 2010, 37:2315-2327. doi:10.1007/s00259-010-1533-y.

16. He J, Ackerly T, O'Keefe GJ, Geso M: Respiratory motion gating based on list-mode data in 3D PET: a simulation study using the dynamic NCAT Phantom. In Proceedings of the 2009 First IEEE International Conference on Information Science and Engineering. Nanjing: IEEE; 2009:3697-3700.

17. Schleyer PJ, O'Doherty MJ, Barrington SF, Marsden PK: Retrospective datadriven respiratory gating for PET/CT. Phys Med Biol 2009, 54:1935-1950. doi:S0031-9155(09)87068-1.

18. Kesner $A L$, Kuntner $C:$ A new fast and fully automated software based algorithm for extracting respiratory signal from raw PET data and its comparison to other methods. Medical Physics 2010, 37:5550-5559.

19. Kesner AL, Bundschuh RA, Detorie NC, Dahlbom M, Ziegler SI, Czernin J, Silverman DH: Respiratory gated PET derived in a fully automated manner from raw PET data. IEEE Trans NuCl Sci 2009, 56:677-686.

20. Hosseini S, Tran I, Botkin C, Mohiuddin A, Nguyen N, Osman M: Respiratory gating in FDG PET/CT: an initial experience [abstract]. J Nucl Med 2009, 50:2219.

21. Mitsumoto K, Abe K, Sakaguchi Y, Zhang T, Tachiya Y, Ohya N, Baba S, Sasaki M: Determination of the optimal acquisition protocol of breathhold PET/CT for the diagnosis of thoracic lesions. Nucl Med Commun 2011, 32:1148-1154. doi:10.1097/MNM.0b013e32834bbda7.

22. Dawood M, Buther F, Jiang X, Schafers KP: Respiratory motion correction in 3-D PET data with advanced optical flow algorithms. IEEE Trans Med Imaging 2008, 27:1164-1175. doi:10.1109/TMI.2008.918321.

23. Bai W, Brady M: Regularized B-spline deformable registration for respiratory motion correction in PET images. Phys Med Biol 2009 54:2719-2736. doi:S0031-9155(09)88487-X.

24. Gigengack F, Ruthotto L, Burger M, Wolters C, Jiang X, Schafers K: Motion correction in dual gated cardiac PET using mass-preserving image registration. IEEE Trans Med Imaging 2011. doi:10.1109/TMI.2011.2175402.

25. Zaidi H: Quantitative Analysis in Nuclear Medicine Imaging. New York: Springer; 2006

26. Christensen GE, Geng X, Kuhl JG, Bruss J, Grabowski Thomas J, Pirwani IA, Vannier MW, Allen JS, Damasio H: Introduction to the non-rigid image registration evaluation project (NIREP). In Proceedings of the Third international conference on Biomedical Image Registration. Utrecht, The Netherlands: Springer; 2006.

27. Miller TR, Sampathkumaran KS: Digital filtering in nuclear medicine. J NuCl Med 1982, 23:66-72.
28. King MA, Miller TR: Use of a nonstationary temporal Wiener filter in nuclear medicine. Eur J Nucl Med 1985, 10:458-461.

29. Kesner, Adam, Dahlbom, Magnus, Czernin, Johannes, Silverman, Daniel: To gate or not to gate: Improved combined respiratory gated images through bootstrapped voxel based noise evaluation. J Nucl Med Meeting Abstracts 2008, 49: 59P-C.

30. Ilovich O, Abourbeh G, Bocher M, Freedman N, Billauer H, Dotan S, Danenberg HD, Mishani E: Structure-activity relationship and preclinical evaluation of carbon-11-labeled ammonium salts as PET-myocardial perfusion imaging agents. Mol Imaging Biol 2012, 14(5):625-636. doi:10.1007/s11307-011-0539-6.

31. Höglund J, Shirvan A, Antoni G, Gustavsson S, Långström B, Ringheim A, Sörensen J, Ben-Ami M, Ziv I: 18F-ML-10, a PET tracer for apoptosis: first human study. J NuCl Med 2011, 52:720-725. doi:110.081786.

32. Bundschuh RA, Martinez-Moeller A, Essler M, Martinez MJ, Nekolla SG, Ziegler SI, Schwaiger M: Postacquisition detection of tumor motion in the lung and upper abdomen using list-mode PET data: a feasibility study. J Nucl Med 2007, 48:758-763.

33. Daube-Witherspoon ME, Muehllehner G: Treatment of axial data in three-dimensional PET. J Nucl Med 1987, 28:1717-1724.

34. Nehmeh SA, Erdi YE, Ling CC, Rosenzweig KE, Schoder H, Larson SM, Macapinlac HA, Squire OD, Humm JL: Effect of respiratory gating on quantifying PET images of lung cancer. J Nucl Med 2002, 43:876-881.

doi:10.1186/2191-219X-3-29

Cite this article as: Kesner et al:: Gating, enhanced gating, and beyond: information utilization strategies for motion management, applied to preclinical PET. EJNMMI Research 2013 3:29.

\section{Submit your manuscript to a SpringerOpen ${ }^{\odot}$ journal and benefit from:}

- Convenient online submission

- Rigorous peer review

- Immediate publication on acceptance

- Open access: articles freely available online

- High visibility within the field

- Retaining the copyright to your article

Submit your next manuscript at $>$ springeropen.com 